\title{
ON PARALLEL HYPERSURFACES IN THE ELLIPTIC AND HYPERBOLIC $n$-DIMENSIONAL SPACE
}

\section{A. SANTAL 6}

1. Introduction. Let $S^{n-1}$ be a hypersurface of class $C^{3}$ in the elliptic or hyperbolic $n$-dimensional space, which is closed and bounding and whose principal curvatures with respect to an inside normal are all positive. Let $S^{n-1}(\lambda)$ be the hypersurface parallel to $S^{n-1}$ at distance $\lambda$.

If $\rho_{1}, \rho_{2}, \cdots, \rho_{n-1}$ are the principal radii of curvature of $S^{n-1}$ at a point $P$ and $d P$ denotes the element of area at $P$, the mean curvatures of $S^{n-1}$ are defined by

$$
M_{i}=\int_{S^{n-1}}\left(\sum \frac{1}{\rho_{r_{1}} \rho_{r_{2}} \cdots \rho_{r_{i}}}\right) d P, \quad i=0,1, \cdots, n-1,
$$

where the sum is extended to the $C_{n-1, i}$ combinations of $i$ th order of the indices $1,2, \cdots, n-1$. In particular, $M_{0}$ coincides with the area $A$ of $S^{n-1}$.

Herglotz [6 $]^{1}$ and, from a more general point of view, Allendoerfer [1] have obtained the area $A(\lambda)$ and volume $V(\lambda)$ of the parallel hypersurface $S^{n-1}(\lambda)$, which can be expressed as linear functions of the mean curvatures $M_{i}$ of $S^{n-1}$ with coefficients depending upon $\lambda$. For this purpose it is enough to find the expression of $A(\lambda)$, that is, $M_{0}(\lambda)$, because $V(\lambda)$ is then given by

$$
V(\lambda)=V+\int_{0}^{\lambda} A(\lambda) d \lambda .
$$

The purpose of the present note is to extend these results to the evaluation of all mean curvatures $M_{i}(\lambda)$ of $S^{n-1}(\lambda)$. The resulting formulae are also linear with respect to $M_{i}$; they are (2.9) for the elliptic case, and (3.2) for the hyperbolic case. For $i=0$, they give the value of $A(\lambda)$ obtained by Herglotz and Allendoerfer.

As a consequence, in the elliptic case we obtain the relation (4.2) between the mean curvature of an $S^{n-1}$ and those of its polar hypersurface. Finally we obtain the equations (5.3) which hold for the mean curvatures of convex surfaces of "constant width" in the elliptic or hyperbolic $n$-dimensional spaces.

In all these questions, in order to obtain simplifications in the re-

Received by the editors January 8, 1949.

1 Numbers in brackets refer to the bibliography at the end of the paper. 
sulting formulas the generalized Gauss-Bonnet formula as obtained by Allendoerfer-Weil [2] plays a fundamental role. In our particular case of the elliptic and hyperbolic space, this formula can be written (see [1]):

For $n-1$ even

$$
C_{n-1} M_{n-1}+C_{n-3} M_{n-3}+\cdots+C_{1} M_{1}+K^{n / 2} V=-\omega^{n} \chi^{\prime} / 2
$$

and for $n-1$ odd

$$
C_{n-1} M_{n-1}+C_{n-3} M_{n-3}+\cdots+C_{0} M_{0}=\omega^{n} \chi^{\prime} / 2
$$

where $\omega^{j}$ is the surface area of a $j$-dimensional unit sphere $\left(\omega^{0}=2\right)$ and

$$
C_{i}=\frac{\omega^{n}}{\omega^{i} \omega^{n-1-i}} K^{(n-1-i) / 2},
$$

being $K=1$ in the elliptic and $K=-1$ in the hyperbolic case. $\chi^{\prime}$ is the inner characteristic of the volume bounded by $S^{n-1}$; if $S^{n-1}$ is a topologic sphere it is $\chi^{\prime}=-1$ for $n-1$ even and $\chi^{\prime}=1$ for $n-1$ odd.

2. The elliptic case. Let $C_{i}(i=1,2, \cdots, n-1)$ be the lines of curvature of $S^{n-1}$ which pass through the point $P$ and let $d s_{i}$ be the element of arc of $C_{i}$ at $P$. The element of area of $S^{n-1}$ at $P$ will be

$$
d P=d s_{1} d s_{2} \cdots d s_{n-1} \text {. }
$$

If $\rho_{i}$ is the principal radius of curvature at $P$ corresponding to $C_{i}$ and $R_{i}$ represents the distance from $P$ to the contact point of the normal to $S^{n-1}$ at $P$ with the envelope of the normals to $S^{n-1}$ along $C_{i}$, the relation (see, for instance, $[5$, p. 214])

$$
\rho_{i}=\tan R_{i}
$$

is well known.

Furthermore if $d \alpha_{i}$ is the angle between two infinitely near normals to $S^{n-1}$ along $C_{i}$ at their intersection point,

$$
d s_{i}=\sin R_{i} d \alpha_{i} .
$$

From (2.1) and (2.3) we deduce

$$
d P=\prod_{i=1}^{n-1} \sin R_{i} d \alpha_{i}
$$

Applying (2.4) to the hypersurface $S^{n-1}(\lambda)$, we have

$$
d P(\lambda)=\prod_{i=1}^{n-1} \sin \left(R_{i}+\lambda\right) d \alpha_{i}
$$


or, according to (2.4),

$$
\begin{aligned}
d P(\lambda) & =\prod_{i=1}^{n-1}\left(\sin R_{i} \cos \lambda+\cos R_{i} \sin \lambda\right) d \alpha_{i} \\
& =\prod_{i=1}^{n-1}\left(\cos \lambda+\sin \lambda / \tan R_{i}\right) d P .
\end{aligned}
$$

From the definition (1.1) and from (2.2) we deduce

(2.7) $M_{i}(\lambda)=\int_{S^{n-1}(\lambda)}\left(\sum \frac{1}{\tan \left(R_{\nu_{1}}+\lambda\right) \cdots \tan \left(R_{v_{i}}+\lambda\right)}\right) d P(\lambda)$,

or, according to (2.5) and (2.4)

$$
\begin{aligned}
& M_{i}(\lambda)=\int \sum\left(\prod_{j=1}^{i} \cos \left(R_{v_{j}}+\lambda\right)\right. \\
&\left.\cdot \prod_{j=i+1}^{n-1} \sin \left(R_{r_{j}}+\lambda\right)\right) d \alpha_{1} \cdots d \alpha_{n-1} \\
&=\int_{S_{n}-1} \sum\left(\prod_{j=1}^{i}\left(\frac{\cos \lambda}{\tan R_{r_{j}}}-\sin \lambda\right)\right. \\
&\left.\cdot \prod_{j=i+1}^{n-1}\left(\cos \lambda+\frac{\sin \lambda}{\tan R_{v_{j}}}\right)\right) d P .
\end{aligned}
$$

The sums are always extended over all combinations of $i$ th order of the indices $1,2, \cdots, n-1$.

If we take into account (2.2) and the definition (1.1) of $M_{i}$, from the last equality results ${ }^{2}$

$$
M_{i}(\lambda)=\sum_{k=0}^{n-1} M_{k} \phi_{i k}(\lambda)
$$

where

(2.10) $\phi_{i k}(\lambda)=\sum_{h=p}^{q}(-1)^{i-h} C_{n-1-k, i-h} C_{k, h} \sin ^{i+k-2 h} \lambda \cos ^{n-1-i-h+2 h} \lambda$,

where the sum is extended over all values of $h$ for which the combina-

2 The combinatory coefficients which appear in (2.10) are easily obtained if we observe that the number of terms in the sum (2.8) with $k$ factors $1 / \tan R_{\boldsymbol{v}_{j}}$ and coefficient $\sin ^{i+k-2 h \lambda} \cos ^{n-1-i-k+2 h} \lambda$ is $C_{i, h} C_{n-1-i, k-k} C_{n-1, i}$ and the number of terms in the sum (1.1) which gives $M_{k}$ is $C_{n-1, k}$. Therefore the product $M_{k} \sin ^{i+k-2 h \lambda} \cos ^{n-1-i-k+2 h} \lambda$ appears a number of times equal to the quotient of the two foregoing combinatory numbers, which is equal to $C_{n-1-k, i-k} C_{k, \Lambda}$. 
tory symbols have a sense, that is

$$
p=\max (0, i+k-n+1), \quad q=\min (i, k) .
$$

Formulas (2.9) and (2.10) solve our problem for the elliptic case.

3. The hyperbolic case. For the case of a hypersurface $S^{n-1}$ in the hyperbolic $n$-dimensional space, formulas (2.2) and (2.3) must be replaced respectively by

$$
\rho_{i}=\tanh R_{i}, \quad d s_{i}=\sinh R_{i} d \alpha_{i} .
$$

Exactly the same calculation as before gives now

$$
M_{i}(\lambda)=\sum_{k=0}^{n-1} M_{k} \phi_{i k}(\lambda)
$$

with

(3.3) $\phi_{i k}(\lambda)=\sum_{h=p}^{q}(-1)^{i-h} C_{n-1-k, i-h} C_{k, h} \sinh ^{i+k-2 h} \lambda \cosh ^{n-1-i-k+2 h} \lambda$, where $p, q$ are given by (2.11).

4. Polar surfaces. In the elliptic case it is interesting to consider the polar surface $S^{n-1}(\pi / 2)$ to the given $S^{n-1}$.

Applying (2.9), (2.10) for $\lambda=\pi / 2$ we obtain

$$
M_{i}(\pi / 2)=(-1)^{i} M_{n-1-i} \text {. }
$$

If $M_{i}^{P}$ denotes the $i$ th mean curvature of the polar surface, we have $M_{i}^{P}=(-1)^{i} M_{i}(\pi / 2)$ and consequently

$$
M_{i}^{P}=M_{n-1-i}
$$

For $i=0$

$$
A^{P}=M_{n-1},
$$

which is a result due to Allendoerfer [1, formula (30)]. For $n=3$ we get $A^{P}=M_{2}, M_{1}^{P}=M_{1}$ or, applying the Gauss-Bonnet formula (1.3)

$$
M_{1}^{P}=M_{1}, \quad A^{P}+A=-4 \pi \chi^{\prime} .
$$

The last formula is due to Blaschke [4].

5. Hypersurfaces of constant width. Let us assume $S^{n-1}$ to be a topological sphere such that the inward drawn normal at every point $P$ cuts $S^{n-1}$ beside $P$ at only one opposite point $P^{*}$. Let $\Delta$ be the distance $P P^{*}$ measured along the normal. If $\Delta$ is constant for every point 
$P, S^{n-1}$ is said to be a hypersurface of "constant width."

In such a case the normal at $P^{*}$ coincides with $P^{*} P$. Indeed, if $Q$ is a point of $S^{n-1}$ such that the distance $P Q$ is a maximum ( $P$ fixed, $Q$ variable on $S^{n-1}$ ), $Q P$ must be normal to $S^{n-1}$ at $Q$ and therefore, by assumption, distance $Q P=\Delta$; on the other hand, if $P^{*} P$ were not normal to $S^{n-1}$ at $P^{*}$, the distance $P P^{*}$ would not be a maximum, thus distance $P P^{*}<$ distance $P Q=\Delta$, contrary to the assumption.

Furthermore, according to the definition of the radii $R_{i}$ and the assumption that they are not negative (see $\$ 1$ and (2.2), (3.1)), the point of contact of the normal $P P^{*}$ with the envelope of the normals along each line of curvature through $P$ does lie inside the segment $P P^{*}$; therefore for the hypersurfaces of constant width, between the corresponding radii $R_{i}, R_{i}^{*}$ at opposite points, the relation

$$
R_{i}+R_{i}^{*}=\Delta, \quad i=1,2, \cdots, n-1,
$$

holds.

We have also $d P=(-1)^{n-1} d P^{*}$, and consequently (2.7) gives

$$
M_{i}(-\Delta)=(-1)^{n-1-i} M_{i}
$$

which holds the same in both elliptic and hyperbolic cases.

Therefore, taking into account the relations (2.9) and (3.2) we get:

Between the mean curvatures $M_{i}$ of a hypersurface of constant width $\Delta$ in the elliptic or hyperbolic n-dimensional space, the relations

$$
M_{i}=(-1)^{n-1-i} \sum_{k=0}^{n-1} M_{k} \phi_{i k}(-\Delta), \quad i=0,1,2, \cdots, n-1,
$$

hold, where $\phi_{i k}$ are given by (2.10) in the elliptic case and by (3.3) in the hyperbolic case.

Furthermore, if $V$ is the volume enclosed by $S^{n-1}$, we have $V(-\Delta)$ $=(-1)^{n} V$ and therefore (1.2) and (2.9), (3.2) give the following relation

$$
V=(-1)^{n} V+(-1)^{n} \sum_{k=0}^{n-1} M_{k} \int_{0}^{-\Delta} \phi_{0 k}(\lambda) d \lambda,
$$

which must be added to the preceding ones (5.3).

The obtained relations (5.3) and (5.4) are, in general, not independent, as the following examples will show.

EXAmple 1. If $n=2,(5.3)$ and (5.4) are equivalent to the unique relation

$$
M_{0} \sin \Delta-M_{1}(1-\cos \Delta)=0 \text { (elliptic case), }
$$




$$
M_{0} \sinh \Delta-M_{1}(1-\cosh \Delta)=0 \text { (hyperbolic case). }
$$

If $L$ is the length and $A$ the area enclosed by $S^{1}, M_{0}=L$ and the Gauss-Bonnet formula gives $M_{1}=2 \pi \pm A$; therefore the foregoing relations may be written respectively

$$
L=(2 \pi-A) \tan (\Delta / 2), \quad L=(2 \pi+A) \tanh (\Delta / 2) .
$$

Example 2. For $n=3$, if we set $M_{0}=A$ and take into account (1.3) which gives $M_{2}=4 \pi \pm A$, the relations (5.3) become equivalent to

$$
\begin{aligned}
M_{1} \cos \Delta & =2(2 \pi-A) \sin \Delta \text { (elliptic case), } \\
M_{1} \cosh \Delta & =2(2 \pi+A) \sinh \Delta \text { (hyperbolic case). }
\end{aligned}
$$

(5.4) gives

$2 V=2 \pi \Delta-\left(M_{1} / 2\right) \sin ^{2} \Delta-(2 \pi-A) \sin \Delta \cos \Delta$ (elliptic case), $2 V=-2 \pi \Delta-\left(M_{1} / 2\right) \sinh ^{2} \Delta+(2 \pi+A) \sinh \Delta \cosh \Delta$ (hyperbolic case).

If we take into account (5.6), the last relations can be written respectively

$$
4 V=4 \pi \Delta-M_{1}, \quad 4 V=M_{1}-4 \pi \Delta .
$$

(5.5) and (5.7) are due to Blaschke [3]. For the analogous questions in the $n$-dimensional euclidean space, see [7].

\section{BIBLIOGRAPHY}

1. C. B. Allendoerfer, Steiner formulae on a general $S^{n+1}$, Bull. Amer. Math. Soc. vol. 54 (1948) pp. 128-135.

2. C. B. Allendoerfer and A. Weil, The Gauss-Bonnet theorem for Riemannian polyhedra, Trans. Amer. Math. Soc. vol. 53 (1943) pp. 101-129.

3. W. Blaschke, Einige Bemerkungen ueber Kurven und Flächen von konstanter Breite, Leipziger Berichte vol. 67 (1915) pp. 290-297.

4. - Integralgeometrie 22: Zur elliptischen Geometrie, Math. Zeit. vol. 41 (1936) pp. 785-786.

5. E. P. Eisenhart, Riemannian geometry, Princeton, 1926.

6. G. Herglotz, Ueber die Steinersche Formel filr Parallelfidchen, Abh. Math. Sem. Hansischen Univ. vol. 15 (1943) pp. 165-177.

7. L. A. Santal6, Sobre los cuerpos convexos de anchura constante en $E_{n}$, Portugaliae Mathematica vol. 5 (1946) pp. 195-201.

The Institute for Advanced Study and

Facultad de Ciencias Matematicas, Rosario, Argentina 